\title{
Electrical and dielectric properties of kevlar - carbon hybrid fiber / epoxy laminated composites
}

\author{
Harith I. Jafer*
}

\author{
Farah T. Mohammed Noori* \\ Ekram A. Al-ajaj*
}

Received 14, March, 2009

Accepted 24, November, 2009

\begin{abstract}
:
This paper reports a.c., d.c. conductivity and dielectric behavior of Ep-hybrid composite with12 Vol.\% Kevlar-Carbon hybrid. D.C. conductivity measurements are conducted on the graded composites by using an electrometer over the temperature range from (293-413) K. It was shown then that conductivity increases by increasing number of Kevlar -Carbon fiber layers (Ep1, Ep2, Ep3), due to the high electrical conductivity of Carbon fiber. To identify the mechanism governing the conduction, the activation energies at low temperature region (LTR) and at high temperature region (HTR) have been calculated. The activation energy values for hybrid composite decrease with increasing number of fiber layers. The a.c. conductivity was measured over frequency range $100 \mathrm{~Hz}-1 \mathrm{MHz}$. It was found that $\sigma(\omega)$ values increase with increasing frequency according to the relation $\sigma(\omega)=\mathrm{Aw}^{\mathrm{s}}$. The values of frequency exponent (s) were found to increase with number of layers.
\end{abstract}

Key words: Conductive fiber, Epoxy resin, Electrical conductivity, KevlarCarbon fiber.

\section{Introduction:}

Epoxies are widely used in insulation, such as electrical machinery, switchgear and bushings in transformers [1]. Polymers have a very low concentration of free charge carriers, and thus are non conductive and transparent to electromagnetic radiation. Due to this reason they are not suitable for use as enclosures for electronic equipment because they cannot shield it from outside radiation. Also they cannot prevent the escape of radiation from the component. Polymers cannot provide protection against electrostatic discharge in handling sensitive electronic Devices [2]. It is known that properties of a composite are depending on properties of its parts. Several fillers can be added to the insulating polymeric matrix in order to achieve different conductivity ranges. Filled epoxy polymer are required for a variety of industrial applications such as electrical, concept of optoelectronic and electronic devices protect electrical components from short circuiting, dust and moisture. In the electronics industry epoxy resins are the primary resin used in over molding integrated circuits, transistors and hybrid circuits, and making printed circuit boards [3, 4]. In this paper, the hybrid composite with 1, 2, 3 layers of $12 \mathrm{vol} \%$ of woven Kevlar-carbon fabric which presents the advantage of lower price and because of kevlar's presence the mechanical properties are improved have been incorporated into epoxy resin and ensuring the reinforcementmatrix is changing the basic electric properties of epoxy matrix. Kevlar

*Department of Physics, College of Science, University of Baghdad, Iraq 
fibers are dielectric while carbon fibers have high electric conductivity so the mixed fabric is actually a superposition of two nets. In such conditions the composite's properties have to be effect of two behaviors. Direct conductivity (d.c). and alternating conductivity (a.c), dielectric constant, dissipation factor $(\tan \delta)$ and relaxation time $(\tau)$ have been determined at different temperatures and frequencies analyzed .The frequency utilized in the study was from $100 \mathrm{~Hz}$ to $1 \mathrm{MHz}$.

\section{D.C Resistivity measurements}

Electrical conductivity is defined as the proportional factor between the current and the electron field. Electrical conductivity of a material is a property with highest variations in values. (N).

For measurement of electrical resistance of insulating materials, three electrodes cell or (guard ring electrode method) was used to study the effect of the filler addition and the temperature on volume resistivity of polymer composite[5].

Resistivity $(\rho)$ and d.c. conductivity ( $\sigma$ d.c.) values were calculated by using the following relation

$\rho=\mathrm{RA} / \ell$

where:

$\rho$ is the resistivity (in units of $\Omega \cdot m$ ) of the material

$\mathrm{R}$ is the resistance of the object, measured in Ohms, equivalent to $\mathrm{J} \cdot \mathrm{s} / \mathrm{C} 2$

$\mathrm{A}$ is the cross sectional area (in units of $\mathrm{m}^{2}$ ), and

$\ell$ is the length (in units of meters)

Resistivity is a measure of the material's ability to oppose electric current. where Conductivity was calculated by using the following formula

$\sigma_{\mathrm{d} \cdot \mathrm{c} \cdot}=1 / \rho \ldots \ldots(2)$
The values of activation energies have been calculated from using the equation:

$\sigma_{\mathrm{d} \cdot c . .}=\sigma_{\mathrm{o}} \exp (-\mathrm{Ea} / \mathrm{kT}) \ldots . .(3)$

where:

$\sigma_{0}$ is the minimum electrical conductivity at $0 \mathrm{~K}$ (ohm.cm) $)^{-1}$.

$\mathrm{Ea}$ is the activation energy

$\mathrm{K}$ is the Boltzmann constant

$\mathrm{T}$ is the temperature

The electrical conductivity is a function of temperature, if the increasing of temperature causes an increasing in free change carries, then the electrical conductivity increases. The rate of activation depends on the number of molecules or particles having sufficient thermal energy to reach the energy barrier and on the velocity of these molecules or atoms that can cross this energy. When the conductivity $\sigma$ is measured at a wide of temperature, Ea can be calculated by plotting $\sigma$ against absolute temperature to give a straight line as equation 3 .

\subsection{A.C measurements}

Ac measurements

are complementary to $\mathrm{dc}$ measurements and not a substitute for them. Alternative current response as a function of frequency offers valuable additional information about the dynamic response of the system. However the principle strength of acstudies lies their ability to provide information on the polarization response A common feature for a.c measurements is that a.c electrical conductivity $\sigma_{a . c}(\omega)$ increased when the frequency is increased, according to the equations below [6]:

$\sigma_{\mathrm{a} . \mathrm{c}}(\omega)=\sigma_{\text {total }}(\omega)-\sigma_{\mathrm{d} . \mathrm{c}}$

$\sigma_{\text {d.c }}$ is the d.c conductivity which depends strongly on temperature and dominate at low frequencies and high temperature, while $\sigma_{a . c}$ is the a.c conductivity which is weaker 
temperature dependence than $\sigma_{\text {d.c }}$ and dominate at high frequency and low temperature then, the The total conductivity was calculated from the equation:

$\sigma_{\text {total }}(\omega)=(\mathrm{d} / \mathrm{A}) \mathrm{G}$

Where $d$ is the thickness of the measured sample, $\mathrm{G}$ is the sample conductance, $\omega$ is the angular frequency $(\mathrm{w}=2 \pi \mathrm{f})$, and $\mathrm{A}$ is the cross sectional area.

The dielectric constant is calculated according to the following equation:

$\varepsilon=\mathrm{C} / \mathrm{C}$ 。

where $\mathrm{C}$ the capacitance of the electrodes with dielectric, $\mathrm{C}_{0}$ is the geometrical capacitance of the sample without dielectric $\left(\mathrm{C}_{\circ}=\varepsilon^{\circ} \mathrm{A} / \mathrm{d}\right.$, where $\varepsilon^{\circ}$ is the permittivity of free space which is equal to $8.85 \times 10^{-12} \mathrm{~F} / \mathrm{m}$ ).

The dielectric loss $\dot{\varepsilon}$ was calculated from the following equation:-

$\dot{\varepsilon}=\mathrm{G} / \omega \mathrm{C}_{0}$

The value of dissipation factor $(\tan \delta)$ can be calculated from the equation:-

$\tan \delta=\dot{\varepsilon} / \varepsilon$

The relaxation time $\tau$ is given by the following equation

$\frac{1}{\omega \tan \delta}=\tau \ldots . .(9)$

\section{Experimental Details:-}

\section{Materials and testing procedure}

The materials used to prepare the test samples were epoxy resin (EPI0 Conbextra) with the hardener aliphatic amine (Hy 956) reinforced by one type of_interplay hybrids (Kevlar -Carbon) woven table (1) show some properties of woven[5]. In order to obtain a composite, interplay hybrids a filler 1, 2, and 3 layers are added to epoxy resin with (12 vol. \%). Hand lay out method was used to prepare specimens with dimensions $100 \times 100 \times 3 \mathrm{~mm}$. Table (2) list the epoxy sample and their composites details

Table(1) properties of Kevlar Carbon woven

\begin{tabular}{|c|c|c|}
\hline Properties & Carbon fiber & $\begin{array}{c}\text { Kavler } \\
\text { fiber(49) }\end{array}$ \\
\hline Diameter & $7-9.7$ & 11.9 \\
\hline Density $\left(10^{3} \mathrm{Kg} / \mathrm{m}^{3}\right)$ & 1.79 & 1.45 \\
\hline Moduulus $(\mathrm{GPa})$ & 228 & 125 \\
\hline $\begin{array}{c}\text { Tensile } \\
\text { Strength }(\mathrm{MPa})\end{array}$ & 3800 & 3000 \\
\hline
\end{tabular}

Table(1)Epoxy and composites

\begin{tabular}{|c|c|}
\hline Specimen & Details \\
\hline EP & Epoxy \\
\hline EP1 & Epoxy reinforced one layer of fiber \\
\hline EP2 & Epoxy reinforced two layer of fiber \\
\hline EP3 & Epoxy reinforced three layer of fiber \\
\hline
\end{tabular}

\section{D.C Resistivity measurements}

D.C conductivity of a dielectric material can be determined as a function of temperature and by using the circuit diagram shown in Fig.(1) The electrical contacts are made of fine copper wires soldered to $\mathrm{Al}$ by indium electrodes using low power soldering iron(30watt). Samples with dimensions $(2 \times 2 \times 0.3) \mathrm{cm}$ were mounted in regulator oven and then resistance were recorded against the temperature raising of the oven over the temperature (293-413) $\mathrm{K}$ by using digital electrometer Keithley (616).

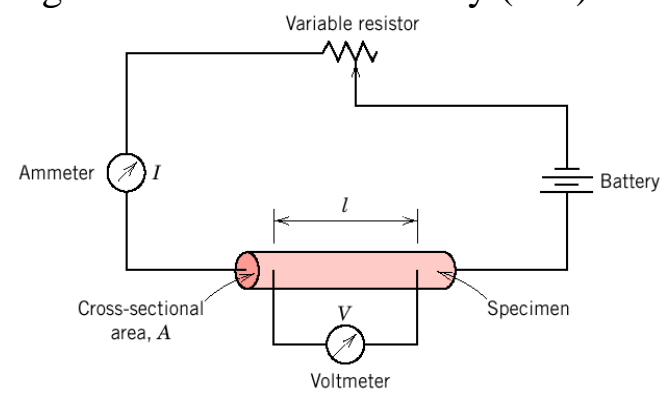

Fig.(1) circuit for measuring d.c conductivity 


\section{A.C measurements}

The electrical properties of the specimens $(2 \times 2 \times 0.3) \mathrm{cm}$ were measured by using automatic LRC bridge (PM60304 Philips) were to measure the sample conductance $\mathrm{G}$ and the capacitance $\mathrm{C}$ directly . The measurement was carried at frequencies from $100 \mathrm{~Hz}$ to $1 \mathrm{MHz}$ at room temperature.

\section{Result and Discussion:}

\section{D.C conductivity:}

The values of D.C conductivity were plotted as a function of temperature (293-413) $\mathrm{K}$ as shown in figure (2). It has been observed that d.c. conductivity increases for all sample with the increase number of layers ( 1 , 2 and 3 ) of kavler-carbon fiber.

In this situation the electrical properties of carbon fiber were almost dominated, since a network may start to connect filler particles to each other and a new kinetic path may be formed. It has been observed that d.c. conductivity suddenly increases after $333 \mathrm{~K}$ in all the samples. This is because $\mathrm{Tg}$ (glass translation temperature) epoxy is around $333 \mathrm{~K}$ [7]. below $\mathrm{Tg}$ d.c. conductivity does not increase much, the amorphous phase of epoxy after $\mathrm{Tg}$ where comes in sudden change in conductivity occurs. This is because after $\mathrm{Tg}$ free volume increases and polymer backbone (main chain) start moving, which makes the movement of molecular chains easy and hence they take place in conduction process with increase temperature. The values of electrical conductivity for epoxy and composites at room temperature given in table (3).

Table(3)The values of $\sigma_{d \cdot c}$ for Epoxy and composites

\begin{tabular}{|c|c|}
\hline Specimen & $\boldsymbol{\sigma}_{\mathbf{d} \cdot \mathbf{c} \cdot}(\mathbf{o h m} . \mathbf{c m})^{-1}$ \\
\hline EP & $1.33^{*} 10^{-9}$ \\
\hline EP1 & $1.45^{*} 10^{-9}$ \\
\hline EP2 & $1.67 * 10^{-9}$ \\
\hline EP3 & $2 * 10^{-9}$ \\
\hline
\end{tabular}

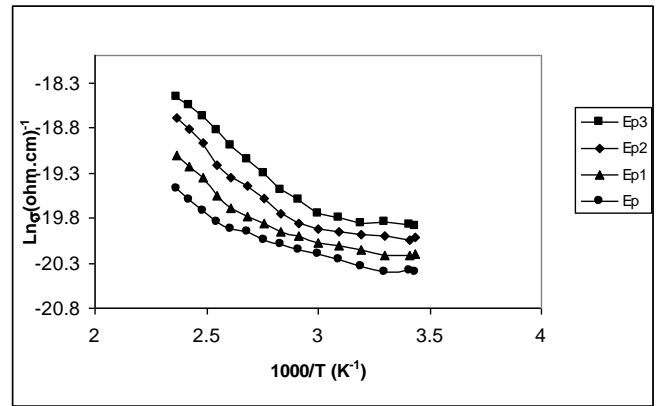

Fig.(2)Temperature dependence of the d.c. electrical conductivity for Ep-hybrid composites with Kevlar carbon

At any temperature only a fraction of molecules or atoms in the system will have sufficient energy to reach activation energy level of Ea. As the temperature of the system is increased, more and more molecules or atoms will attain the activation energy level. Values of the activation energy (Ea) were calculated for all the investigated composites from the slopes of the linear lines of $\ln \sigma$ versus 1000/T according to equation (3). It is observed that at high temperature, the activation energy of dipole segmental processes decreases due to disturbance of the co-operative movement of segments [8]. This explains the decrease of activation energy with increase the number of layer as shown in table (2), it is found that there are two stages of conductivity throughout the heating temperature range. The first activation energy $\left(E_{a 1}\right)$ occurs at high temperatures within the range (333$373) \mathrm{K}$ and it is due to conduction of the carrier excited into the extended states beyond the mobility edge. While the second activation energy $\left(\mathrm{E}_{\mathrm{a} 2}\right)$ occurs at low temperatures within the range $(293-328) \mathrm{K}$ and the conduction mechanism of this stage is due to carriers transport to localized states near the valence and conduction bands. These two conduction mechanism means that the d.c. conductivity is non linear with temperature. 
Table (2): The data of activation energy of neat and reinforced epoxy with Kevlar-Carbon

\begin{tabular}{|l|l|l|}
\hline Specimens & $\mathrm{E}_{\mathrm{av} 2}$ & $\mathrm{E}_{\mathrm{av} 1}$ \\
\hline EP & 0.745 & 0.0906 \\
\hline EP1 & 0.189 & 0.065 \\
\hline EP2 & 0.141 & 0.059 \\
\hline EP3 & 0.112 & 0.037 \\
\hline
\end{tabular}

\section{Ac Conductivity:}

Figure (3) shows the ac electrical conductivity of epoxy composite with 1, 2, 3 layers of 12 vol. \% of woven Kevlar-carbon in epoxy matrix. The ac conductivity increases with increasing the frequency applied in the experiment according to the relation $\sigma(\omega)=A w^{s}$ where, $\mathrm{A}$ is constant, and $(\mathrm{s})$ is a frequency exponent. The rise of conductivity upon increasing the frequency is a common respond for polymeric and semiconductor samples is due to ions motion and the transport near or at the interstitial surface between fiber and epoxy network, a.c conductivity can be explain in terms of the hopping of charge carries conduction mechanism (CBH)[9] and each number of kevlar-carbon layer made a capacitance cooperation in conduction process which polarization (dipole formation/orientation) and is described by $\mathrm{P}=\mathrm{Q}^{\prime} / \mathrm{A}$ is responsible for the increase in charge density. To support this conclusion frequency exponent (s) has been observed and is determined from the slope of a plot Ln $\sigma_{\text {a.c }}(\omega)$ versus $\operatorname{Ln} \omega$, then (s) is not constant but usually $0<<1$ [6].

On the other hand figure (4) shown that the exponent factor (s) was decreased with increasing the number of layers of Kevlar-carbon in epoxy. It is observed that the values are less than unity (0.648-0.403), another indication for correlated barrier hopping $(\mathrm{CBH})$ mechanism [10].

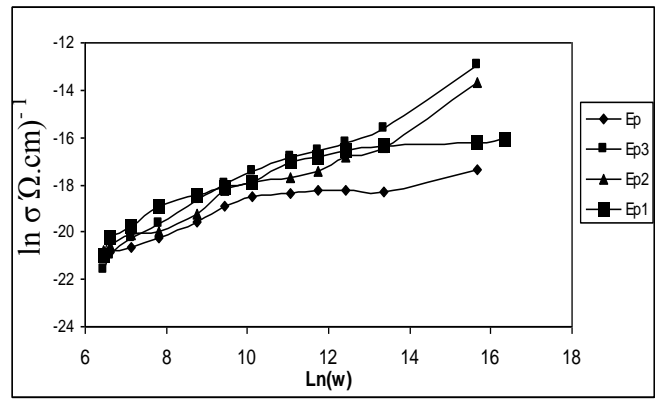

Fig.(3)Frequency dependence of the a.c. electrical conductivity for Ephybrid composites with Kevlar Carbon / epoxy matrix

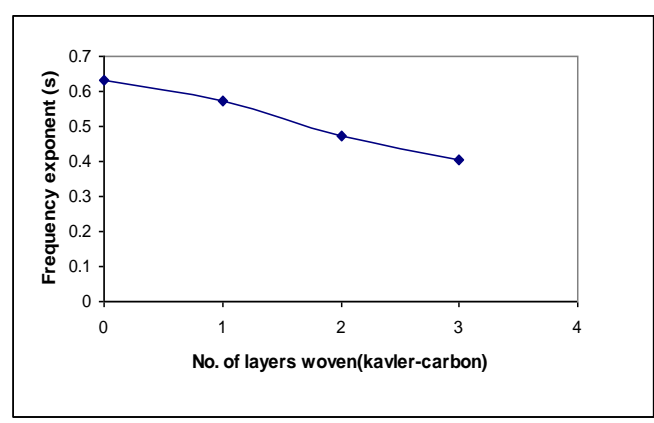

Fig.(4) Frequency exponent dependence on the number of layers of Ep-hybrid composites with Kevlar - Carbon

\section{Dielectric Behavior:}

It is observed from figure (5) that for the entire sample, the dielectric constants increase with the increase number of layers. Also the figure shows that the dielectric constant decreased with increasing frequency and reaches a constant value for all samples at room temperature due to the interfacial polarization only. The decrease of $\varepsilon$ with increasing frequency is the expected behavior in most dielectric materials This is due to dielectric relaxation involves the orientation polarization which in turn depends upon the molecular arrangement of dielectric to be material .So, at higher frequencies, the rotational motion of the polar molecules of dielectric is not sufficiently rapid for the attainment of equilibrium with the field, hence dielectric constant seems to be 
decreasing with increasing frequency. In general, the higher value of dielectric constant at low frequencies for materials can be attributed to the fact that the electrode blocking layers is dominated mechanism at low frequencies region[11].

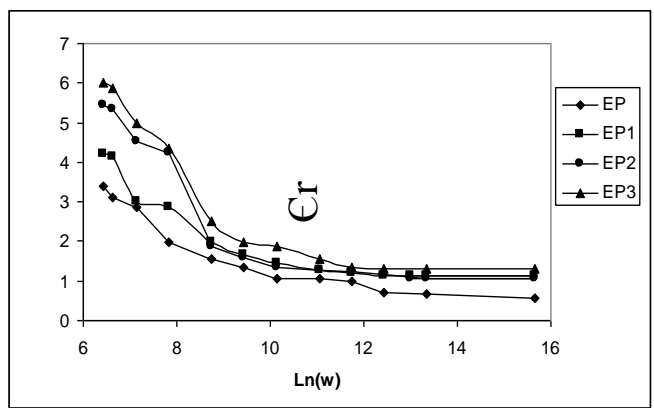

Fig.(5) Frequency dependence of dielectric constant for Ep- hybrid composites with Kevlar - Carbon fiber

Fig. 6 shows the variation of dielectric loss with frequency for hybrid composites with Kevlar- carbon fiber epoxy matrix for different layers at room temperatures. The loss spectra characterized by peak appearing at a characteristic frequency for samples suggest the presence of relaxing dipoles in all the samples. The strength and frequency of relaxation depend on characteristic property of dipolar relaxation. The tangent loss peaks shift towards the higher frequency side [12].

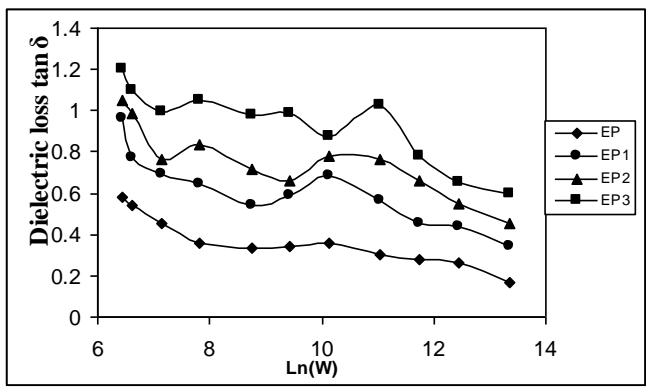

Fig.(6) Frequency dependence of dielectric loss for Ep-hybrid composites with Kevlar - Carbon fiber filled epoxy matrix
Fig. (7) show the relaxation time with frequency, from figure was found the variation are decrease with increase number of layers of Kevlar-carbon fiber in epoxy matrix.

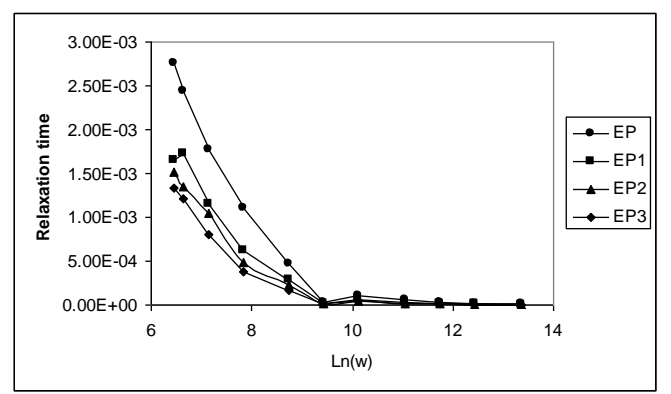

Fig.(7) Frequency dependence of relaxation time for hybrid composites with Kevlar - Carbon fiber filled epoxy matrix

\section{Conclusion:}

(1) Increase number of layers of Kevlar-carbon fiber content increases the a.c. and d.c. conductivity value.

(2) D.C. conductivity of Kevlar-carbon hybrid fiber reinforced epoxy resin increased with increase of temperature. After $60^{\circ} \mathrm{C}(333) \mathrm{K}$ there is a sudden increase in d.c. Conductivity due to Tg.

(3) Activation energy decreases with increase of fiber.

(4) Frequency exponent(s) decreases with increase of fiber.

(5) The dielectric constant decreases with the increase in frequency.

\section{References:}

1- Zhou1 Y. X., Wu2 P. X., Cheng2, Z-Y., Ingram1 J.and, Jeelani1 S. 2008. Improvement in electrical, thermal and mechanical properties of epoxy by filling carbon nanotube "Express Polymer Letters 2(1): 40-48.

2-Navin Chand* and Deepak Jain 2004. "Evaluation of a.c. conductivity behaviour of graphite filled polysulphide modified epoxy composites". Bull. Mater. Sci., 27(3): 227-233. 
3-Neelakanta, P. S. 1995. "Handbook of electromagnetic materials", (Boca Raton: CRC Press) p. 7.

4- Chung D. D. L. Chung and Shoukai Wang 1999. "Carbon fiber polymer- matrix structural composite as a semiconductor and concept of optoelectronic and electronic devices made from it" Smart Mater. Struct. 8(2): 161-166.

5-Cîrciumaru, A., Andrei, G., Bîrsan, I.-G.. 2007." Electric and electromagnetic Proceeding of the $1^{\text {st }}$ International Conference on Polymers Properties of Fiber Fabric Based Filled Epoxy Composites Processing in Engineering”, Galați, : 212-222.

6-Donnely, K.P. and Varlow, B.R.. 2003. "Nonlinear dc and ac conductivity in electrically insulating composites Dielectric and Electric Insulation". 10():610614.

7- Chand N. and Nigrawal A. 2007 " Investigations on d.c. conductivity behavior of milled carbon fiber reinforced epoxy graded composites" Bull. Mater. Sci., 30(2): 167-172.

8-Dillip K., Choudhary R.N.P, and samantaray B.K. 2008. "Study of dielectric relaxation and a.c. conductivity behavior of plasticized polymer nanocomposite electrolyte" online publisher.

9-B.K.Choi, Y.W. Kim and K. H. Shin, j.Powder sources, 199768.

10-Liu Y. and Gong A. 2006. "Compressive Behavior and Energy Absorption of etal Porous Polymer Composite with Interpentrating Network Structure "Transctions of Nonferrous Metal Society of Ching. 1(6): 439-443.

11-Abbas R. A. 2007."Studing Some Dielectric Properties and Effective Parameters of Composite Materials Containing of Novolak Resin" ,Journal of Engineering and Technology , 25(8):277-288.

12-Tsangaris G.M., Psarras G.C. and A.J. $\quad$ Kontopoulos. 2003. "Dielectric permittivity and loss of an aluminum-filled epoxy resin " Zographou, 15773 Athens, Greece Available online 25 April.

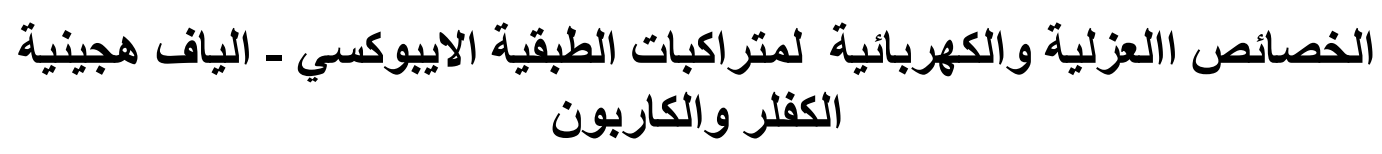

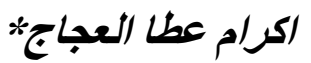

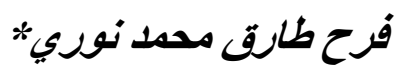

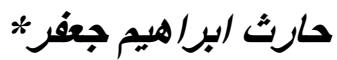

*امعة بغداد ـ كلية العلوم - قسم الفيزياء

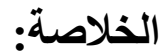

تم في هذا البحث در اسة التوصيلية المتناوبة والمستمرة وسلوك العزل للمتر اكب الهجيني المكون من الاييوكسي

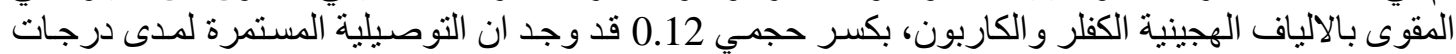

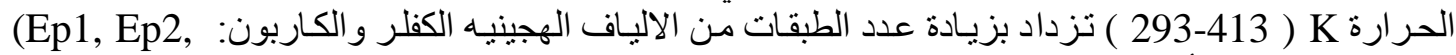
بp3)

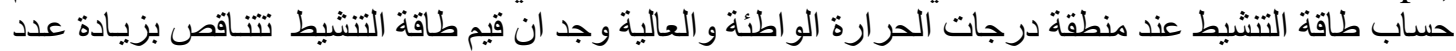

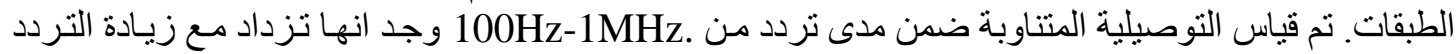
على وفق العلاقة 